\title{
WDVV equations and invariant bi-Hamiltonian formalism
}

\author{
J. Vašíček ${ }^{a, b}$ and R. Vitolo ${ }^{b, c}$ \\ ${ }^{a}$ Mathematical Institute of the Silesian University, Silesian University, \\ Opava, Czech Republic \\ ${ }^{b}$ Department of Mathematics and Physics "E. De Giorgi", University of Salento, \\ Lecce, Italy \\ ${ }^{c}$ Istituto Nazionale di Fisica Nucleare - Sezione di Lecce, \\ Lecce, Italy \\ E-mail: jakub.vasicek@math.slu.cz, raffaele.vitolo@unisalento.it
}

ABSTRACT: The purpose of the paper is to show that, in low dimensions, the WDVV equations are bi-Hamiltonian. The invariance of the bi-Hamiltonian formalism is proved for $N=3$. More examples in higher dimensions show that the result might hold in general. The invariance group of the bi-Hamiltonian pairs that we find for WDVV equations is the group of projective transformations. The significance of projective invariance of WDVV equations is discussed in detail. The computer algebra programs that were used for calculations throughout the paper are provided in a GitHub repository.

KEYwords: Integrable Hierarchies, Topological Field Theories, Differential and Algebraic Geometry, Field Theories in Lower Dimensions

ARXIV EPRINT: 2104.13206 


\section{Contents}

1 Introduction 1

2 Preliminaries: Hamiltonian operators $\quad 8$

$\begin{array}{lll}3 & \text { WDVV equations as systems of conservation laws } & 10\end{array}$

4 Bi-Hamiltonian formalism for WDVV equations, $N=3 \quad 13$

$\begin{array}{lll}4.1 & \text { B.A. Dubrovin's normal forms of } \eta & 13\end{array}$

$\begin{array}{lll}4.2 & \text { O.I. Mokhov and N.A. Pavlenko's normal forms of } \eta & 15\end{array}$

$\begin{array}{ll}4.3 \text { An example: equation of flat centroaffine metrics } & 17\end{array}$

$\begin{array}{lll}4.4 & \text { Remarks on the generic case } & 18\end{array}$

5 WDVV equations in higher dimensions $\quad 19$

$\begin{array}{ll}5.1 \text { The case } N=4 & 19\end{array}$

$\begin{array}{ll}5.2 \text { The case } N=5 & 21\end{array}$

6 Conclusions: projective geometry of WDVV systems 22

\section{Introduction}

The Witten-Dijkgraaf-Verlinde-Verlinde (WDVV) equations are an overdetermined system of Partial Differential Equations (PDEs) that originated in two-dimensional topological field theory $[17,68]$. Since then, they have been a central subject in Theoretical Physics, with applications ranging from supersymmetric quantum mechanics [5, 37, 46], topological quantum field theory [39], string theory $[10,18]$ and supersymmetric gauge theory [40].

In nearly all the above research subjects, WDVV equations are associated with integrable systems. The deep connections between these two fields were first described in the seminal work of B.A. Dubrovin [24]. Since then, WDVV equations continue to attract the attention of Mathematicians. Few recent works show the depth and breadth of their investigation $[9,12,16,36,59-62,69]$.

The aim of this paper is to present new results on the Hamiltonian formalism for WDVV equations and related geometric properties, and eventually stimulate further research along this direction in the field.

The Hamiltonian formalism for PDEs has been developed for more than 50 years with the idea to reproduce geometric structures and mathematical results analogous to those of integrable systems in Hamiltonian mechanics (see, e.g., [52]).

The main difficulty in bringing the Hamiltonian formalism to the WDVV equations is that the Hamiltonian formalism was developed for evolution PDEs, while WDVV equations 
are an overdetermined system of PDEs in a single unknown function. More precisely, an evolutionary system of PDEs of the form

$$
u_{t}^{i}=f^{i}\left(u^{j}, u_{x}^{j}, u_{x x}^{j}, \ldots\right), \quad i=1, \ldots, n
$$

in $n$ unknown functions of two independent variables $u^{i}=u^{i}(t, x)$ is said to be Hamiltonian if there exists a linear differential operator $A=A^{i j \sigma} \partial_{\sigma}$, where $A^{i j \sigma}=A^{i j \sigma}\left(u^{k}, u_{x}^{k}, u_{x x}^{k}, \ldots\right)$ and $\partial_{\sigma}=\partial_{x} \circ \cdots \circ \partial_{x}$ ( $\sigma$-times), and a density $H=\int h d x$, where $h=h\left(u^{k}, u_{x}^{k}, u_{x x}^{k}, \ldots\right)$ such that

$$
u_{t}^{i}=f^{i}\left(u^{j}, u_{x}^{j}, u_{x x}^{j}, \ldots\right)=A^{i j \sigma} \partial_{\sigma} \frac{\delta H}{\delta u^{j}} .
$$

$H$ is said to be a Hamiltonian density. The operator $A$ is required to define a Poisson bracket between conserved densities $F, G$ of the PDE:

$$
\{F, G\}_{A}=\int \frac{\delta F}{\delta u^{i}} A^{i j \sigma} \partial_{\sigma} \frac{\delta G}{\delta u^{j}} d x .
$$

The skew-symmetry of the Poisson bracket is equivalent to the skew-adjointness of the operator: $A^{*}=-A$, and the Jacobi identity is equivalent to the vanishing of the Schouten bracket of the operator: $[A, A]=0$ (see, e.g. $[19,38,47,49]$ ). An operator $A$ fulfilling the above properties is said to be a Hamiltonian operator.

A bi-Hamiltonian system of PDEs is just a system of PDEs that is Hamiltonian with respect to two operators $A_{1}, A_{2}$ (and the respective Hamiltonian densities). The operators are required to be compatible: their Schouten bracket vanishes $\left[A_{1}, A_{2}\right]=0$, or the pencil $A_{1}+\lambda A_{2}$ is a Hamiltonian operator for every $\lambda \in \mathbb{R}$. In this case, Magri's Theorem [48] yields an infinite sequence of commuting conserved quantities or symmetries, which is usually identified with integrability.

In order to see how to transform the WDVV system into an evolutionary system, we shall first recall the basic notions. We will follow [25]. The mathematical problem is: in $\mathbb{R}^{N}$ find a function $F=F\left(t^{1}, \ldots, t^{N}\right)$ such that

1. $\frac{\partial^{3} F}{\partial t^{1} \partial t^{\alpha} \partial t^{\beta}}=\eta_{\alpha \beta}$ is a constant symmetric nondegenerate matrix;

2. $c_{\alpha \beta}^{\gamma}=\eta^{\gamma \epsilon} \frac{\partial^{3} F}{\partial t^{\epsilon} \partial t^{\alpha} \partial t^{\beta}}$ are the structure constants of an associative algebra;

3. $F$ is quasihomogeneous: $F\left(c^{d_{1}} t^{1}, \ldots, c^{d_{N}} t^{N}\right)=c^{d_{F}} F\left(t^{1}, \ldots, t^{N}\right)$.

If $e_{1}, \ldots, e_{N}$ is the basis of $\mathbb{R}^{N}$ then the algebra operation is $e_{\alpha} \cdot e_{\beta}=c_{\alpha \beta}^{\gamma}(\mathbf{t}) e_{\gamma}$ with unity $e_{1}$. The WDVV, or associativity, system of PDEs takes the form

$$
\eta^{\mu \lambda} \frac{\partial^{3} F}{\partial t^{\lambda} \partial t^{\alpha} \partial t^{\beta}} \frac{\partial^{3} F}{\partial t^{\nu} \partial t^{\mu} \partial t^{\gamma}}=\eta^{\mu \lambda} \frac{\partial^{3} F}{\partial t^{\nu} \partial t^{\alpha} \partial t^{\mu}} \frac{\partial^{3} F}{\partial t^{\lambda} \partial t^{\beta} \partial t^{\gamma}} .
$$

The unknown of the system is not exactly $F$, as the above requirements completely specify the functional dependence from $t^{1}$ (up to a second degree polynomial, see [25]):

$$
F=\frac{1}{6} \eta_{11}\left(t^{1}\right)^{3}+\frac{1}{2} \sum_{k>1} \eta_{1 k} t^{k}\left(t^{1}\right)^{2}+\frac{1}{2} \sum_{k, s>1} \eta_{s k} t^{s} t^{k} t^{1}+f\left(t^{2}, \ldots, t^{N}\right) .
$$


This implies that the WDVV system is an overdetermined system in one unknown function $f$ of $N-1$ independent variables. Just as an example, in the case $N=3$ we have a single equation on $f=f\left(t^{2}, t^{3}\right)=f(x, t)$. It was proved in [24] that, when $\eta_{11}=0$, the matrix $\eta_{\alpha \beta}$ can be transformed by a linear change of coordinates that preserves $\partial / \partial t^{1}$ to

$$
\eta_{\alpha \beta}=\delta_{\alpha+\beta, N+1}=\left(\begin{array}{lll}
0 & 0 & 1 \\
0 & 1 & 0 \\
1 & 0 & 0
\end{array}\right),
$$

and the WDVV equation becomes

$$
f_{t t t}=f_{x x t}^{2}-f_{x x x} f_{x t t} .
$$

A technique was developed by O. Mokhov [50] in order to rewrite the WDVV equation in the case $N=3$ as a first-order quasilinear system, or hydrodynamic-type system, of PDEs. Namely, if we introduce coordinates $a=f_{x x x}, b=f_{x x t}, c=f_{x t t}$ then for (1.7) we have the compatibility conditions

$$
\left\{\begin{array}{l}
a_{t}=b_{x}, \\
b_{t}=c_{x}, \\
c_{t}=\left(b^{2}-a c\right)_{x}
\end{array}\right.
$$

We will say that the above system is a first-order WDVV system. The above system is of the general conservative first-order quasilinear form

$$
u_{t}^{i}=\left(V^{i}(\mathbf{u})\right)_{x}=\frac{\partial V^{i}}{\partial u^{j}} u_{x}^{j},
$$

where $u^{i}=u^{i}(t, x)$ are field variables, $i=1, \ldots, n$. The above representation allowed to find a bi-Hamiltonian formalism for the equation (1.7) [29]:

$$
u_{t}^{i}=A_{1}^{i j} \frac{\delta H_{2}}{\delta u^{j}}=A_{2}^{i j} \frac{\delta H_{1}}{\delta u^{j}} .
$$

with respect to two compatible local Hamiltonian operators $A_{1}$ and $A_{2}$, with expressions

$$
\begin{aligned}
A_{1} & =\left(\begin{array}{ccc}
-\frac{3}{2} \partial_{x} & \frac{1}{2} \partial_{x} a & \partial_{x} b \\
\frac{1}{2} a \partial_{x} & \frac{1}{2}\left(\partial_{x} b+b \partial_{x}\right) & \frac{3}{2} c \partial_{x}+c_{x} \\
b \partial_{x} & \frac{3}{2} \partial_{x} c-c_{x} & \left(b^{2}-a c\right) \partial_{x}+\partial_{x}\left(b^{2}-a c\right)
\end{array}\right), \\
A_{2} & =\left(\begin{array}{ccc}
0 & 0 & \partial_{x}^{3} \\
0 & \partial_{x}^{3} & -\partial_{x}^{2} a \partial_{x} \\
\partial_{x}^{3}-\partial_{x} a \partial_{x}^{2} & \partial_{x}^{2} b \partial_{x}+\partial_{x} b \partial_{x}^{2}+\partial_{x} a \partial_{x} a \partial_{x}
\end{array}\right) .
\end{aligned}
$$

The above Hamiltonian operators are homogeneous with respect to the grading $\operatorname{deg} \partial_{x}=$ 1. The Hamiltonian densities are $H_{2}=\int c d x$ (for $\left.A_{1}\right)$ and $H_{1}=\int\left[-(1 / 2) a\left(\partial_{x}^{-1} b\right)^{2}-\right.$ $\left.\left(\partial_{x}^{-1} b\right)\left(\partial_{x}^{-1} c\right)\right] d x$ (for $A_{2}$; this one is nonlocal). It should be remarked that B. Dubrovin proved that WDVV equations are integrable by providing a Lax pair for arbitrary $N$ [24]. Nonetheless, knowing the bi-Hamiltonian formalism for a system of PDEs is an additional 
source of information. In the WDVV case, it will be shown here that the additional information is provided by the invariance properties of the bi-Hamiltonian structure.

First-order homogeneous Hamiltonian operators (HHOs) were introduced in [26]. They have the form

$$
A_{1}^{i j}=g^{i j} \partial_{x}+\Gamma_{k}^{i j} u_{x}^{k}
$$

where $g^{i j}=g^{i j}(\mathbf{u})$ transforms as a symmetric contravariant tensor (we will always assume that $\operatorname{det}\left(g^{i j}\right) \neq 0$ ) whose inverse $g_{i j}$ is a flat pseudo-Riemannian metric with Christoffel symbols $\Gamma_{j k}^{i}=-g_{j s} \Gamma_{k}^{s i}$.

It should be stressed that solutions of WDVV equations yield Frobenius manifolds, or integrable hierarchies of PDEs defined by bi-Hamiltonian pairs of first-order HHOs (see, e.g., [21, 22, 24]). However, the above quasilinear system of first-order PDEs (1.8) is exceptional with respect to the theory of Frobenius manifolds as it is bi-Hamiltonian with respect to a pair of a first-order HHO and a third-order HHO. Higher order homogeneous Hamiltonian operators were introduced in [23], and have a considerably more complicated structure than (1.12). Third-order HHOs can always be transformed to the canonical form

$$
A_{2}=\partial_{x}\left(h^{i j} \partial_{x}+c_{k}^{i j} u_{x}^{k}\right) \partial_{x}
$$

(again, we require that the leading coefficient is non-degenerate: $\left.\operatorname{det}\left(h^{i j}\right) \neq 0\right)[7,20,57,58]$ which is invariant with respect to the action of projective reciprocal transformations [33, 34] (see section 2 for more details).

In further papers it was shown that a bi-Hamiltonian formulation as above exists for a different choice of the matrix $\eta_{i j}$ (in the case $N=3$ ) [41] or after the exchange of $t$ and $x$ in (1.7) [42], and much more recently, in the case $N=4$ for $\eta^{(1)}$ (1.14) [55]. In a new interesting paper [51], the classification of $N=3$ WDVV equations admitting a Hamiltonian formalism with a local first-order HHO as in (1.11) was given.

It was natural to try to prove that WDVV equations admit a bi-Hamiltonian formulation by means of a compatible pair of a first-order HHO and a third-order HHO for any choice of $\eta$. The idea for the proof is first to prove that the invariance group of WDVV equations, i.e. linear transformations in the space $\left(t^{1}, \ldots, t^{N}\right)$ that leave $\partial / \partial t^{1}$ invariant [25], do not change the form of a bi-Hamiltonian pair as above. Then, it is enough to prove the statement only on normal forms with respect to the invariance group.

Indeed, using the invariance group of the problem, one can reduce the matrix $\eta_{i j}$ to two canonical forms if the quasihomogeneity weights are distinct:

$\eta_{11}=0:$ the canonical form is $\eta^{(1)}=\left(\eta_{\alpha \beta}^{(1)}\right)$ with

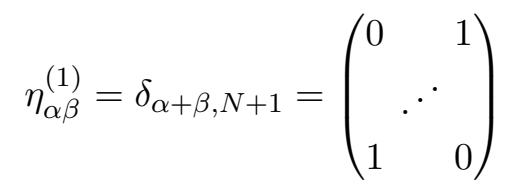

where $F=\frac{1}{2}\left(t^{1}\right)^{2} t^{N}+\frac{1}{2} t^{1} \sum_{\alpha=2}^{N-1} t^{\alpha} t^{N-\alpha+1}+f\left(t^{2}, \ldots, t^{N}\right)$; 
$\eta_{11} \neq 0$ : this case can only happen if $d_{F}=3$, and the canonical form is $\eta^{(2)}=\left(\eta_{\alpha \beta}^{(2)}\right)$ with

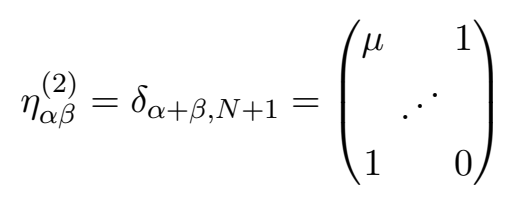

where $\mu \neq 0$ and $F=\frac{\mu}{6}\left(t^{1}\right)^{3}+\frac{1}{2} t^{1} \sum_{\alpha=2}^{N}\left(t^{\alpha}\right)^{2}+f\left(t^{2}, \ldots, t^{N}\right)$.

If one drops the quasihomogeneity request on $F$, a smaller group of linear transformations can be used to show that if $N=3$ then there are 4 distinct canonical forms [51]. The results in [51] also suggested that we should consider a wider class of first-order HHOs, namely the non-local Ferapontov operators (see e.g. [27]). It should be stressed that the necessary theoretical background and software for computations with nonlocal operators was not available until recently $[14,15]$.

That led to the first part of the results in this paper. Namely, in the case $N=3$ we have:

- for any choice of the matrix $\eta$ the first-order WDVV systems admit a third-order homogeneous Hamiltonian operator in canonical form

$$
A_{2}=\partial_{x}\left(h^{i j} \partial_{x}+c_{k}^{i j} u_{x}^{k}\right) \partial_{x}
$$

- the first-order WDVV systems defined by matrices $\eta$ in the orbit of $\eta^{(1)}$ admit a first-order local homogeneous Hamiltonian operator of the type

$$
A_{1}^{i j}=g^{i j} \partial_{x}+\Gamma_{k}^{i j} u_{x}^{k}
$$

- the first-order WDVV systems defined by matrices $\eta$ in the orbit of $\eta^{(2)}$ admit a first-order non-local homogeneous Hamiltonian operator of Ferapontov type

$$
\begin{aligned}
A_{1}^{i j}= & g^{i j} \partial_{x}+\Gamma_{k}^{i j} u_{x}^{k}+\alpha V_{q}^{i} u_{x}^{q} \partial_{x}^{-1} V_{p}^{j} u_{x}^{p} \\
& +\beta\left(V_{q}^{i} u_{x}^{q} \partial_{x}^{-1} u_{x}^{j}+u_{x}^{i} \partial_{x}^{-1} V_{q}^{j} u_{x}^{q}\right)+\gamma u_{x}^{i} \partial_{x}^{-1} u_{x}^{j}
\end{aligned}
$$

where $V_{j}^{i}=\partial V^{i} / \partial u^{j}$ is the matrix of velocities of the first-order WDVV system (1.9) and $\alpha, \beta, \gamma$ are three constants;

- finally, first-order WDVV systems are bi-Hamiltonian: the Schouten bracket of the two operators vanishes, $\left[A_{1}, A_{2}\right]=0$, or the pencil $A_{1}+\lambda A_{2}$ is a Hamiltonian operator for every $\lambda \in \mathbb{R}$.

- We also realized that the quasihomogeneity of $F$ in the assumptions on the WDVV problem (item 3 on page 2) can be dropped without changing all our results in the above items. Indeed, in a recent paper [51] Mokhov and Pavlenko classified the WDVV equations without the requirement of quasihomogeneity of the solutions, and obtained 4 canonical forms. For all of them we recover the bi-Hamiltonian pair, as we will show in section 4.2 . 
The above results imply that in the case $N=3$ the WDVV quasilinear first-order systems are linearly degenerate, non diagonalizable and in the Temple class, as it follows from the main results in [35]. Indeed, the presence of third-order operators yields many interesting properties of the underlying first-order quasilinear system of PDEs.

In higher dimensions proving a general invariance theorem is more difficult, and will be considered in the future. However, if $N=4$ and $\eta=\eta^{(1)}$ it was already known that the first-order WDVV system had a first-order local HHO [31] and a compatible third-order HHO [55]. In this paper we prove that

- if $N=4$ and $\eta=\eta^{(2)}$ then the first-order WDVV system admits a third-order HHO of the form (1.13);

- if $N=5$ and $\eta=\eta^{(1)}$ or $\eta=\eta^{(2)}$ (with $\mu=1$, as the case with an arbitrary $\mu \neq 0$ was beyond the capabilities of our servers), then the first-order WDVV systems admit a third-order HHO of the form (1.13).

We did not try to find the first-order operator in the case $N=4$ and $\eta=\eta^{(2)}$ or when $N=5$ : indeed, the results in [11] that we used to find the first-order operators in the case $N=3$ do not hold when $N \geq 4$.

Even if invariance is fully stated only in the case $N=3$, the presence of first-order and third-order HHOs in higher dimensions is enough to support the following conjecture.

Conjecture. The WDVV equations in the form of quasilinear systems of first-order PDEs are bi-Hamiltonian with respect to a pair of a third-order HHO in canonical form (1.13) and a first-order HHO, which can either be local (1.12) in the case $\eta=\eta^{(1)}$, or nonlocal of general Ferapontov type

$$
A_{1}^{i j}=g^{i j}(\mathbf{u}) \partial_{x}+\Gamma_{k}^{i j}(\mathbf{u}) u_{x}^{k}+\sum_{\alpha} c^{\alpha \beta} w_{\alpha k}^{i}(\mathbf{u}) u_{x}^{k} \partial_{x}^{-1} w_{\beta h}^{j}(\mathbf{u}) u_{x}^{h},
$$

where $c^{\alpha \beta}$ is a constant symmetric matrix, in the case $\eta=\eta^{(2)}$.

There are interesting implications of the conjecture. Indeed, it was proved in [33, 35] that third-order HHOs can be regarded as distinguished projective varieties, namely, quadratic line complexes. They determine families of varieties, linear line congruences, that correspond to first-order quasilinear systems of PDEs (first-order WDVV being one such systems in all known cases).

As the above correspondence between integrable systems and projective varieties is non-standard, it is instructive to show it in the simplest example of WDVV equation (1.7). We will use the form (1.8). The leading coefficient $\bar{g}=\left(g^{i j}\right)$ of $A_{2}$ in (1.11b):

$$
\left(g^{i j}\right)=\left(\begin{array}{ccc}
0 & 0 & 1 \\
0 & 1 & -a \\
1 & -a & 2 b+a^{2}
\end{array}\right)
$$

has the inverse matrix $g=\bar{g}^{(-1)}$ that is a Monge metric. It can be written in the form

$$
g=-2 b d a^{2}+2 a d a d b+2 d a d c+d b^{2} .
$$


Now, it is easy to realize that the above metric represents the equation of a quadratic line complex in the space of lines of the projective space $\mathbb{P}^{3}$. Indeed, considering two infinitesimally close points in a 4-dimensional projective space $P=\left[u^{0}, u^{1}, u^{2}, u^{3}\right]$ and $P+d P=\left(u^{0}+d u^{0}, u^{1}+d u^{1}, u^{2}+d u^{2}, u^{3}+d u^{3}\right)$, the minors of the matrix $(P, P+d P)$ turn out to be of the form $p^{i j}=u^{i} d u^{j}-u^{j} d u^{i}$ (note that $u^{0}=1$ and $d u^{0}=0$ when passing to the affine coordinates $a=u^{1}, b=u^{2}, c=u^{3}$ ). Such forms are the Lie form of Plücker coordinates. We recall that the Plücker coordinates characterize lines in $\mathbb{P}^{3}$ modulo the further Plücker relations $p^{i j} p^{h k}+p^{i h} p^{k j}+p^{i k} p^{j h}=0$. We can rewrite $g$ as

$$
g=2(a d b-b d a) d a+2 d a d c+d b^{2} .
$$

The above metric turns out to be a quadratic expression in the Lie form of Plücker coordinates; the corresponding quadratic line complex is given by the system

$$
2 p^{12} p^{01}+2 p^{01} p^{03}+\left(p^{02}\right)^{2}=0, \quad p^{01} p^{23}+p^{02} p^{31}+p^{03} p^{12}=0 .
$$

The line congruence corresponding to the system (1.8) is an $n$-parameter family of lines in $\mathbb{P}^{n+1}$. In homogeneous coordinates $\left[y^{1}, \ldots, y^{n+2}\right]$ it has the general form $y^{i}=u^{i} y^{n+1}+$ $V^{i} y^{n+2}[3,4]$. The lines of the congruence pass through the points $y^{i}=u^{i}, y^{n+1}=1$, $y^{n+2}=0$ and $y^{i}=V^{i}, y^{n+1}=0, y^{n+2}=1$, respectively. The corresponding Plücker coordinates are the minors of the matrix

$$
\left(\begin{array}{ccccc}
u^{1} & \cdots & u^{n} & 1 & 0 \\
V^{1} & \cdots & V^{n} & 1 & 0
\end{array}\right)
$$

The line congruence is linear if there are $n$ linear relations between the Plücker coordinates; these are $n$ linear line complexes. As it was proved in [35], every first-order quasilinear system of PDEs admitting a third-order HHO is associated with a linear line congruence. In the WDVV example (1.8) the linear line congruence takes the form

$$
y^{1}=a y^{4}+b y^{5}, \quad y^{2}=b y^{4}+c y^{5}, \quad y^{3}=c y^{4}+\left(b^{2}-a c\right) y^{5}
$$

The above constructions have a general validity: each time that a third-order HHO in the form (1.13) is found for a system of conservation laws (as we will do many times in the paper) then one can construct the corresponding algebraic varieties.

Then, it turns out that the bi-Hamiltonian pairs and the systems of first-order PDEs are invariant with respect to projective reciprocal transformations. These are non-local (or non-holonomic) transformations of the independent variables of the form

$$
\begin{aligned}
d \tilde{x} & =\left(a_{i} u^{i}+a\right) d x+\left(a_{i} V^{i}+b\right) d t, \\
d \tilde{t} & =\left(b_{i} u^{i}+c\right) d x+\left(b_{i} V^{i}+d\right) d t,
\end{aligned}
$$

which, together with the affine transformation of dependent variables, generate the projective action on the linear line congruence and the quadratic line complex. More in detail, the above transformation can be factorized in a sequence of transformations $R_{1} \circ E \circ R_{2}$ where $E$ is just the exchange of $t$ and $x$ and $R_{i}$ are transformations of the form

$$
d \tilde{x}=\left(a_{i} u^{i}+a\right) d x+\left(a_{i} V^{i}+b\right) d t, \quad d \tilde{t}=d t,
$$

where the dependent variables undergo a projective transformation $\tilde{u}^{i}=\left(A_{j}^{i} u^{i}+A_{0}^{i}\right) /\left(a_{i} u^{i}+\right.$ a) (see [35] for the definition). Hence, the whole bi-Hamiltonian WDVV hierarchy becomes 
a projective-geometric object (in known cases). The above conjecture can be rephrased as follows:

Conjecture. To every WDVV system there are associated a quadratic line complex and a linear line congruence.

This fact might have an (at the moment unpredictable) impact in the applications of WDVV equations. The projective group, in its realization as a group of distinguished reciprocal transformations, is larger than the invariance group of WDVV equations (which is the group of linear transformations leaving $\partial / \partial t^{1}$ invariant, see section 3 ) and its implications in the search for solutions of WDVV equations is still to be understood. Consequences in projective and enumerative geometry are not unlikely. See section 6 for details.

The paper is structured as follows. Section 2 describes the pre-requisites on homogeneous Hamiltonian operators. In section 3 the invariance of bi-Hamiltonian pairs with respect to invariance transformations of WDVV equations is proved. In section 4 biHamiltonian formalism for all normal forms of WDVV equations in the case $N=3$ is provided. Section 5 considers the problem of finding Hamiltonian structures for WDVV in higher dimensions. The concluding section 6 discusses the projective-geometric aspects of the results obtained so far.

The calculations have been done by means of computer algebra systems. In particular, Schouten brackets involving nonlocal operators have been calculated by the Reduce package CDE, and checked by the Maple package jacobi.mpl; both packages are described in [14, 67] (see also [47]). Further calculations have been done in Reduce (finding first-order nonlocal operators when $N=3$, and finding third-order operators in the cases $N=3, N=4$ ) and in Maple, also using the package Jets [8] (finding third-order operators in the case $N=5$ ). The programs are available at a GitHub repository [65].

\section{Preliminaries: Hamiltonian operators}

In this paper we will look for Hamiltonian operators for quasilinear systems of first-order PDEs that are generated by WDVV equations. Known examples suggest that these can be homogeneous operators of first and third order. Let us describe such classes more in detail.

First order local homogeneous operators (1.12) have already been described in the Introduction. We will need their nonlocal generalization (1.19). Such operators were introduced and studied by Ferapontov (see [27]). We will always assume that the leading coefficient is a non-degenerate matrix: $\operatorname{det}\left(g^{i j}\right) \neq 0\left(\right.$ we set $\left.\left(g_{i j}\right)=\left(g^{i j}\right)^{-1}\right)$. It is well known that the Hamiltonian property is equivalent to the following conditions: the symmetry of $g^{i j}$, the fact that $\Gamma_{i k}^{j}=-g_{i p} \Gamma_{k}^{p j}$ are the Christoffel symbols of $g_{i j}$ (interpreted as a pseudoRiemannian metric), and the identities:

$$
\begin{aligned}
g^{i k} w_{\alpha k}^{j} & =g^{j k} w_{\alpha k}^{i}, \\
\nabla_{k} w_{\alpha j}^{i} & =\nabla_{j} w_{\alpha k}^{i}, \\
{\left[w_{\alpha}, w_{\beta}\right] } & =0 \\
R_{k l}^{i j} & =c^{\alpha \beta}\left(w_{\alpha k}^{i} w_{\beta l}^{j}-w_{\alpha k}^{j} w_{\beta l}^{i}\right) .
\end{aligned}
$$


Here, $\nabla$ is the Levi-Civita connection of $g_{i j}, R_{k l}^{i j}=g^{i s} R_{s k l}^{j}$ (we follow the sign conventions of [21]), the bracket $\left[w_{\alpha}, w_{\beta}\right]$ is the usual commutator of the matrices $w_{\alpha}=w_{\alpha k}^{i}$ and $w_{\beta}=w_{\beta k}^{i}$. If the operator is local, then the conditions reduce to those of the local operators (1.17).

Third-order homogeneous Hamiltonian operators are much more complicated in general. However, in the canonical form (1.13) (again, we will assume that the leading coefficient is non-degenerate, $\operatorname{det}\left(h^{i j}\right) \neq 0$, and we set $\left.h_{i j}=\left(h^{i j}\right)^{-1}\right)$ the Hamiltonian property of $A_{2}$ implies that $c_{k}^{i j}$ being given by

$$
c_{s k m}=\frac{1}{3}\left(h_{s m, k}-h_{s k, m}\right),
$$

where $c_{i j k}=h_{i q} h_{j p} c_{k}^{p q}$, so that the leading coefficient determines the operator [33]. The Jacobi property further implies that [33]

$$
\begin{aligned}
h_{m k, s}+h_{k s, m}+h_{m s, k} & =0, \\
c_{m s k, l} & =-h^{p q} c_{p m l} c_{q s k} .
\end{aligned}
$$

The equation (2.3) is equivalent to the fact that $h_{i j}$ is the Monge form, or Monge metric, of a quadratic line complex, a distinguished family of projective varieties. The projective properties of third-order HHOs will be discussed in section 6 .

It is important to remark that $h_{i j}$ turn out to be second degree polynomials with respect to the field variables, under the further algebraic constraints (2.3). A complete classification of operators in the form $(1.13)$ is given in [33,34] for a number of components $n \leq 4$. The classification uses the projective invariance of third-order homogeneous operators with respect to reciprocal transformations of the form (1.27).

We need a way to find Hamiltonian operators for WDVV systems. This is provided by the theory of differential coverings [43]. In our particular case, it is known [63] (but see also [66]) that a necessary condition for $A_{1}$ (both in the local and non-local case) to be the Hamiltonian operator of a quasilinear system of first-order PDEs (1.9) is:

$$
g^{i k} V_{k}^{j}=g^{j k} V_{k}^{i}, \quad \nabla^{i} V_{k}^{j}=\nabla^{j} V_{k}^{i},
$$

where $V_{k}^{i}=\partial V^{i} / \partial u^{k}$ and $\nabla^{j}$ is the Levi-Civita connection of $g_{i j}$. In the case of third-order operators it was recently found [35] that the compatibility conditions between a third-order Hamiltonian operators and a quasilinear system of first-order conservation laws (1.9) are:

$$
\begin{aligned}
h_{i m} V_{j}^{m} & =h_{j m} V_{i}^{m}, \\
c_{m k l} V_{i}^{m}+c_{m i k} V_{l}^{m}+c_{m l i} V_{k}^{m} & =0, \\
h_{k s} V_{i j}^{k} & =c_{s m j} V_{i}^{m}+c_{s m i} V_{j}^{m} .
\end{aligned}
$$

It is interesting to observe that the conditions (2.5) might be relatively difficult to solve for the metric $g^{i j}$, while the system of compatibility conditions for $h^{i j}$, being expressed in lower indices, is indeed a linear algebraic system with respect to the coefficients of the second degree polynomials $h_{i j}$, which is easy to solve. 


\section{WDVV equations as systems of conservation laws}

As we have seen in the Introduction, the WDVV equations (1.4) in the unknown function $f=f\left(t^{2}, \ldots, t^{N}\right)(1.5)$ are presented as a system of conservation laws (1.9) by introducing new dependent variables. The general algorithm for the transformation was first given in [50] in the case $N=3$ and then generalized for arbitrary values of $N$ in [31], the details are exposed below.

\section{Algorithm.}

1. Choose one distinguished independent variable $t^{i}, i>1$ (for example $t^{2}$ ), and all third-order derivatives of $f$ that contain at least one instance of $t^{2}$; call them $u^{1}=$ $f_{t^{2} t^{2} t^{2}}, u^{2}=f_{t^{2} t^{2} t^{3}}, \ldots, u^{n}=f_{t^{2} t^{N} t^{N}}$. These are new dependent variables, and $n=N(N-1) / 2$.

2. Choose another independent variable $t^{j} \neq t^{2}, j>1$ (for example $t^{3}$ ), and, for any $u^{i}$, find $u_{t^{3}}^{i}$ as the $t^{2}$-derivative of an expression $V^{i}$ :

$$
u_{t^{3}}^{i}=V^{i}(\mathbf{u})_{t^{2}} .
$$

There are two possibilities:

(a) either $V^{i}(\mathbf{u})$ is one of the coordinates $u^{k}$, with $k \neq i$;

(b) $V^{i}$ is a third-order derivative of $f$ which is not one of the $u^{k}$. In this case, $V^{i}$ must be expressed by means of one of the equations of the WDVV system. This is always possible due to the structure of the WDVV system.

There are equations in the WDVV system which depend on variables that are not $t^{2}$ or $t^{3}$ derivatives; such equations shall be discarded in the above construction. However, changing the two distinguished independent variables, and using other equations in the WDVV system, one obtains $N-2$ distinct commuting systems of conservation laws with the same structure as above.

In general, first-order WDVV-systems for a fixed (but arbitrary) $N$ and a fixed choice of $\eta$ are provided by the Algorithm as $N-2$ commuting two-dimensional quasilinear firstorder systems of PDEs with $n=N(N-2) / 2$ components [31].

Definition 1. We say that a quasilinear first-order system of conservation laws (3.1) where $\left(u^{i}\right)$ are third-order derivatives of $f$ and the equations are compatibility conditions for a $W D V V$ system to be a first-order WDVV system.

The purpose of this paper is to show that first-order WDVV systems admit a thirdorder HHO in canonical form (1.13) and a compatible first-order local or non-local operator of the type (1.18) for low dimension $N$.

In particular, in the case $N=3$ we will be able to prove that all first-order WDVV systems, i.e. for any choice of matrix $\eta$, admit a bi-Hamiltonian pair as stated above. To this aim, the strategy is: 
1. prove that the invariance group of the WDVV equations do not affect the 'form' of a bi-Hamiltonian pair as above;

2. prove that there is a bi-Hamiltonian pair as above for each canonical form of the WDVV equations, i.e., the canonical form of the matrix $\eta_{i j}$.

The invariance group of the WDVV equations with the quasihomogeneity constraint is the group of linear transformations that preserve the direction of $\partial / \partial t^{1}$ :

$$
\tilde{t}^{\alpha}=P_{\beta}^{\alpha} t^{\beta}+Q^{\alpha}, \quad \operatorname{det}\left(P_{\beta}^{\alpha}\right) \neq 0, \quad P_{1}^{\alpha}=\delta_{1}^{\alpha}
$$

ref. [25]. We have the transformation rules

$$
\frac{\partial}{\partial t^{\alpha}}=P_{\alpha}^{\beta} \frac{\partial}{\partial \tilde{t}^{\beta}}, \quad d \tilde{t}^{\alpha}=P_{\beta}^{\alpha} d t^{\beta},
$$

which imply that the equation (1.4) is transformed into the same equation with respect to the new coordinates $\left(\tilde{t}^{\alpha}\right)$ (of course, one should change coordinates in $F$ and $\eta_{i j}$ ).

Theorem 2. Let $N=3$, and suppose that a WDVV system in first-order form $u_{t}^{i}=$ $\left(V^{i}(\mathbf{u})\right)_{x}$ is bi-Hamiltonian with respect to a pair of compatible Hamiltonian operators $A_{1}$, $A_{2}$, where $A_{1}$ is a nonlocal first-order HHO (1.18) and $A_{2}$ is a local third-order HHO (1.13).

Then, the coordinate change (3.2) does not change the form of the bi-Hamiltonian pair $A_{1}, A_{2}$.

Proof. The matrix $P=\left(P_{\beta}^{\alpha}\right)$ of the change of coordinates can be factorized as

$$
\begin{gathered}
P=T_{1} \cdot T_{2}, \quad \text { where } \\
P=\left(\begin{array}{ccc}
1 & P_{2}^{1} & P_{3}^{1} \\
0 & P_{2}^{2} & P_{3}^{2} \\
0 & P_{2}^{3} & P_{3}^{3}
\end{array}\right), \quad T_{1}=\left(\begin{array}{ccc}
1 & 0 & 0 \\
0 & P_{2}^{2} & P_{3}^{2} \\
0 & P_{4}^{3} & P_{3}^{3}
\end{array}\right), \quad T_{2}=\left(\begin{array}{ccc}
1 & P_{2}^{1} & P_{3}^{1} \\
0 & 1 & 0 \\
0 & 0 & 1
\end{array}\right) .
\end{gathered}
$$

The matrix $T_{1}$ can be further factorized as

$$
\begin{gathered}
T_{1}=R_{1} \cdot E \cdot R_{2}, \quad \text { where } \\
R_{1}=\left(\begin{array}{lll}
1 & 0 & 0 \\
0 & \alpha & \beta \\
0 & 0 & 1
\end{array}\right), \quad E=\left(\begin{array}{lll}
1 & 0 & 0 \\
0 & 0 & 1 \\
0 & 1 & 0
\end{array}\right), \quad R_{2}=\left(\begin{array}{lll}
1 & 0 & 0 \\
0 & \gamma & \delta \\
0 & 0 & 1
\end{array}\right)
\end{gathered}
$$

when $P_{2}^{3} \neq 0$ (when it is zero no factorization is needed).

Let us now choose $t=t^{3}$ and $x=t^{2}$. When $N=3$ there is only one WDVV equation, from which the above Algorithm yields the following system:

$$
\begin{aligned}
& u_{t}^{1}=u_{x}^{2}, \\
& u_{t}^{2}=u_{x}^{3}, \\
& u_{t}^{3}=\phi(\mathbf{u})_{x},
\end{aligned}
$$

where $\phi$ is a rational function of the field variables. 
The transformation $T_{2}$ does not change third-order derivatives of $f$, hence it does not affect the system (3.8).

The transformation $R_{1}$ (equivalently, $R_{2}$ ) has the effect of a reciprocal transformation that preserves the coordinate $t$, namely

$$
d \tilde{x}=P_{2}^{2} d x+P_{3}^{2} d t=\alpha d x+\beta d t, \quad d \tilde{t}=d t .
$$

Such transformations are proved to preserve the canonical form of a third-order HHO [33] and the locality (or the non-local form) of a first-order HHO [32, 54]. At the same time, the third-order derivatives $u^{1}=f_{x x x}, u^{2}=f_{x x t}, u^{3}=f_{x t t}$ undergo the affine transformation:

$$
\begin{aligned}
& u^{1}=\alpha^{3} \tilde{u}^{1}, \\
& u^{2}=\alpha^{2} \beta \tilde{u}^{1}+\alpha^{2} \tilde{u}^{2}, \\
& u^{3}=\alpha \beta^{2} \tilde{u}^{1}+2 \alpha \beta \tilde{u}^{2}+\alpha \tilde{u}^{3},
\end{aligned}
$$

where $\tilde{u}^{1}=f_{\tilde{x} \tilde{x} \tilde{x}}, \tilde{u}^{2}=f_{\tilde{x} \tilde{x} \tilde{x}}, \tilde{u}^{3}=f_{\tilde{x} \tilde{t} \tilde{t}}$. Again, that does not modify the structure of the bi-Hamiltonian pair.

The transformation $E$ is just an exchange of the independent variables. It preserves both the canonical form of the third-order HHO [35] and the locality (or the non-local form) of the first-order HHO. This completes the proof.

Remark 3. In the case $N=3$ after a change of coordinates of the type $R_{1}$ we obtain a new $W D V V$ equation. Hence, we can construct a new quasilinear first-order WDVV system:

$$
\bar{u}_{\bar{t}}^{i}=\left(\bar{V}^{i}(\overline{\mathbf{u}})\right)_{\bar{x}}
$$

using the above Algorithm. However, it can be proved that in general there does not exist an affine transformation that brings the new system into the system $\tilde{u}_{\bar{t}}^{i}=\left(\tilde{V}^{i}(\tilde{\mathbf{u}})\right)_{\tilde{x}}$. Later, the relation between the two systems will be clarified.

Remark 4. In the case $N=3$ the transformation $E$ brings the system (3.8) into the system

$$
\tilde{u}_{\tilde{t}}^{1}=\phi(\tilde{\mathbf{u}})_{\tilde{x}}, \quad \tilde{u}_{\tilde{t}}^{2}=\tilde{u}_{\tilde{x}}^{1}, \tilde{u}_{\tilde{t}}^{3}=\tilde{u}_{\tilde{x}}^{2}
$$

interchanging $\tilde{u}^{1}$ and $\tilde{u}^{3}$ bring the system in the same form as (3.8).

The case $N=4$ cannot be treated in the above way. Indeed, the invariance transformation mix the independent variables, and the commuting systems are transformed in a more complicated way. However, we will be able to show that third-order HHO are present for first-order WDVV systems in canonical forms of WDVV equations when $N=4$ and $N=5$. 


\section{Bi-Hamiltonian formalism for WDVV equations, $N=3$}

Having Theorem 2 at hand, we can investigate $N=3$ WDVV systems and look for biHamiltonian pairs in their representation as quasilinear systems of first-order PDEs. If we prove that if the WDVV system obtained by each canonical form of the matrix $\eta_{i j}$ is endowed with a bi-Hamiltonian pair of the type that we discussed in the Introduction, then this will be true for an arbitrary matrix $\eta_{i j}$.

We will also discuss bi-Hamiltonian pairs for the canonical forms in [51] and for one significant example which comes from centroaffine geometry [28].

While we will look for third-order operators in the canonical form (1.13), we shall explain the reason for choosing the ansatz (1.18) for the first-order operators.

Indeed, computational experiments show that Dubrovin's canonical form $\eta^{(2)}$ does not admit a local first-order HHO. Then, the form (1.18) is the only possibility in the class of Ferapontov operators: the vectors that multiply $\partial_{x}^{-1}$ must be (generalized) commuting symmetries of the quasilinear systems of PDEs in view of the Hamiltonian property of $A_{1}$ [27] (see (2.1)). Now, first-order WDVV systems are non-diagonalizable, as we will discuss in section 6 , and non-diagonalizable systems with a low number of components have only two such symmetries, ${ }^{1}$ namely $\varphi_{1}=u_{x}^{i} \partial / \partial u^{i}$ and $\varphi_{2}=\left(V^{i}\right)_{x} \partial / \partial u^{i}$, which correspond to $t$ and $x$ translational symmetries. This means that the Hamiltonian property for an operator $A_{1}$ of the form (1.18) is equivalent to the conditions (2.1a), (2.1b) and

$$
\begin{aligned}
R_{k l}^{i j}= & \alpha\left(V_{k}^{i} V_{l}^{j}-V_{l}^{i} V_{k}^{j}\right) \\
& +\beta\left(V_{k}^{i} \delta_{l}^{j}-V_{k}^{j} \delta_{l}^{i}-V_{l}^{i} \delta_{k}^{j}+V_{l}^{j} \delta_{k}^{i}\right)+\gamma\left(\delta_{k}^{i} \delta_{l}^{j}-\delta_{l}^{i} \delta_{k}^{j}\right)
\end{aligned}
$$

(obviously, the above two symmetries commute). So, finding operators (1.18) amounts at finding the metric $g^{i j}$ and the three constants $\alpha, \beta, \gamma$.

To this end, we recall a theorem in [11] that states that, for non-diagonalizable hydrodynamic-type systems in $n=3$ unknown functions, the metric of a first-order Hamiltonian operator for the system shall be proportional to a contraction of the square of the Haantjes tensor:

$$
g_{i j}=f H_{i \beta}^{\alpha} H_{j \alpha}^{\beta}, \quad f=f(\mathbf{u}) .
$$

See [11, eq. 2.2 and 2.4] for a coordinate expression of the Nijenhuis and the Haantjes tensors.

Summarizing, in the case $n=3$ there are one unknown function and three unknown constants to be determined in order to find a first-order operator. We will use the above equations in order to determine, by computer algebra, the first-order operator $A_{1}$ for firstorder WDVV systems.

\subsection{B.A. Dubrovin's normal forms of $\eta$}

We start with a result that follows from known calculations.

\footnotetext{
${ }^{1}$ There is only experimental evidence of this fact for $n=3,4,5$ (E.V. Ferapontov, private communication).
} 
Theorem 5. Each first-order WDVV system in the orbit of $\eta^{(1)}$ under the action of the transformations (3.2) is endowed by a compatible pair of local homogeneous Hamiltonian operators $A_{1}$ as in (1.12) and $A_{2}$ as in (1.13).

Proof. The existence of a bi-Hamiltonian pair as in the statement was proved in [29] (see also the Introduction). The fact that the bi-Hamiltonian pair propagates to each element of the orbit is a direct consequence of Theorem 2 .

A completely new result holds for Dubrovin's second canonical form (1.15). We stress that we could obtain this result only for recent developments in the calculations of Schouten brackets for weakly nonlocal operators $[13,14]$.

Theorem 6. Each first-order WDVV system in the orbit of $\eta^{(2)}$ under the action of the transformations (3.2) is endowed by a compatible pair of homogeneous Hamiltonian operators $A_{1}$, a nonlocal operator as in (1.18), and $A_{2}$ as in (1.13).

Proof. If $\eta_{11} \neq 0$, then we have the canonical form

$$
\eta=\left(\begin{array}{lll}
\mu & 0 & 1 \\
0 & 1 & 0 \\
1 & 0 & 0
\end{array}\right)
$$

with $\mu \neq 0$, to which it corresponds the equation

$$
\mu f_{t t t} f_{x x t}-f_{t t t}+\left(f_{x x t}\right)^{2}-f_{x x x} f_{x t t}-\mu\left(f_{x t t}\right)^{2}=0 .
$$

The Algorithm yields the quasilinear first-order system

$$
\begin{aligned}
& a_{t}=b_{x}, \\
& b_{t}=c_{x}, \\
& c_{t}=\left(\frac{a c-b^{2}+\mu c^{2}}{\mu b-1}\right)_{x},
\end{aligned}
$$

where we used the notation $a=f_{x x x}, b=f_{x x t}, c=f_{x t t}$ for the sake of simplicity. We will adopt the same notation throughout the rest of the section.

The above system admits a third-order $\mathrm{HHO} A_{2}$ which is completely determined by the metric

$$
h_{i j}=\left(\begin{array}{ccc}
b(\mu b-2) & (a+\mu c)(1-\mu b) & (\mu b-1)^{2} \\
(a+\mu c)(1-\mu b) & \mu(a+\mu c)^{2}+1 & \mu(a+\mu c)(1-\mu b) \\
(\mu b-1)^{2} & \mu(a+\mu c)(1-\mu b) & \mu(\mu b-1)^{2}
\end{array}\right)
$$

and has the following form:

$$
A_{2}=\left(\begin{array}{ccc}
-\mu \partial_{x}^{3} & 0 & \partial_{x}^{3} \\
0 & \partial_{x}^{3} & \partial_{x}^{2} \frac{a+\mu c}{\mu b-1} \partial_{x} \\
\partial_{x}^{3} & \partial_{x} \frac{a+\mu c}{\mu b-1} \partial_{x}^{2} & \frac{1}{2}\left(\partial_{x}^{2} K \partial_{x}+\partial_{x} K \partial_{x}^{2}\right)
\end{array}\right)
$$


where

$$
K=\frac{(a+\mu c)^{2}+b(2-\mu b)}{(\mu b-1)^{2}} .
$$

We stress that the operator lies in the projective class $g^{(3)}$, according to the classification in $[33,34]$. Systems that possess a third-order Hamiltonian operator have a non-local Hamiltonian that is specified in [35].

Using the results from [11] (see the beginning of the section) we find that the system (4.5) has the first-order operator of Ferapontov type $A_{1}$ defined by the metric

$$
g^{i j}=\left(\begin{array}{ccc}
b^{2} \mu^{2}-a^{2} \mu-2 b \mu-3 & a-a b \mu+b c \mu^{2}-c \mu & 2 b-b^{2} \mu+c^{2} \mu^{2} \\
a-a b \mu+b c \mu^{2}-c \mu & 2 b-b^{2} \mu+c^{2} \mu^{2} & \frac{c\left(a c \mu^{2}-2 b^{2} \mu^{2}+4 b \mu+c^{2} \mu^{3}-3\right)}{b \mu-1} \\
2 b-b^{2} \mu+c^{2} \mu^{2} & \frac{c\left(a c \mu^{2}-2 b^{2} \mu^{2}+4 b \mu+c^{2} \mu^{3}-3\right)}{b \mu-1} & \frac{\delta}{(b \mu-1)^{2}}
\end{array}\right),
$$

where

$$
\begin{aligned}
\delta= & a^{2} c^{2} \mu^{2}-2 a b^{2} c \mu^{2}+4 a b c \mu+2 a c^{3} \mu^{3}-4 a c+b^{4} \mu^{2}-4 b^{3} \mu-3 b^{2} c^{2} \mu^{3} \\
& +4 b^{2}+6 b c^{2} \mu^{2}+c^{4} \mu^{4}-5 c^{2} \mu
\end{aligned}
$$

and the values of constants from (1.18) are $\alpha=-\mu^{2}, \beta=0, \gamma=\mu$.

Using the results from [15] and the module developed in [14] of the software package CDE we are able to prove the compatibility of $A_{1}$ and $A_{2}$ : the Schouten bracket $\left[A_{1}, A_{2}\right]$ vanish.

The fact that the bi-Hamiltonian pair propagates to each element of the orbit is a direct consequence of Theorem 2 .

\subsection{O.I. Mokhov and N.A. Pavlenko's normal forms of $\eta$}

In a recent paper [51] Mokhov and Pavlenko classified the WDVV equations (1.4), without the requirement of quasihomogeneity of the solutions (item 3 in the Introduction), under transformations of the type $T_{1}(3.4)$. They came to the following four canonical forms:

$$
\begin{aligned}
\eta^{1}=\left(\begin{array}{lll}
0 & 0 & 1 \\
0 & \lambda & 0 \\
1 & 0 & \mu
\end{array}\right), & \lambda^{2}=1 ; & \eta^{3}=\left(\begin{array}{lll}
1 & 0 & 1 \\
0 & 0 & 1 \\
1 & 1 & 0
\end{array}\right) ; \\
\eta^{2}=\left(\begin{array}{lll}
1 & 0 & 1 \\
0 & \lambda & 0 \\
1 & 0 & \mu
\end{array}\right), & \lambda^{2}=1 ; & \eta^{4}=\left(\begin{array}{ccc}
1 & 0 & 0 \\
0 & \lambda & 0 \\
0 & 0 & \mu
\end{array}\right), \quad \lambda^{2}=1, \mu^{2}=1 .
\end{aligned}
$$

According to Theorems 5 and 6 , the above examples can be rewritten as bi-Hamiltonian first-order WDVV systems. Let us provide the first-order and third-order HHOs in all the above cases.

The case $\boldsymbol{\eta}^{\mathbf{1}}$. In this case in [51] it is provided a first-order local HHO $A_{1}^{1}$. We look for a third-order one. The WDVV equations in first-order form are of the type (3.8), where the function $\phi$ is defined by

$$
\phi=b^{2}-a c-\lambda \mu b+\mu^{2} .
$$


It is easy to show that the equations (2.6) have the unique solution $h_{i j}$

$$
h_{i j}=\left(\begin{array}{ccc}
-2 b+\lambda \mu & a & 1 \\
a & 1 & 0 \\
1 & 0 & 0
\end{array}\right) .
$$

which is of type $g^{(5)}$ (as (1.8)) according to the classification in [33]. We will omit the corresponding operator $A_{2}^{1}$, as it can be easily reconstructed.

The Schouten bracket $\left[A_{1}^{1}, A_{2}^{1}\right]$ turns out to be zero and thus the above operators are compatible; the computation has been performed by means of the Reduce package CDE, see $[47,67]$.

The case $\boldsymbol{\eta}^{2}$. For $\eta^{2}$ we have the function $\phi(a, b, c)$ :

$$
\phi(a, b, c)=\frac{b^{2}-a c-\lambda c^{2}-\mu b^{2}+\mu a c-\mu \lambda b+\mu^{2}}{1-\lambda b},
$$

for an arbitrary real constant $\mu$.

In this case, the WDVV system in first-order form has a third-order HHO which is completely determined by the metric of type $g^{(3)}[33,34]$ :

$$
h_{i j}=\left(\begin{array}{ccc}
\mu-b(2 \lambda-b) & \frac{(\lambda-b)(\mu a-\lambda c-a)}{\mu-1} & \frac{-(\lambda-b)^{2} \lambda}{\mu-1} \\
\frac{(\lambda-b)(\mu a-\lambda c-a)}{\mu-1} & \frac{(\mu-1)^{2}\left(\lambda+a^{2}\right)-2(\mu-1) \lambda a c+c^{2}}{(\mu-1)^{2}} & \frac{-(\mu a-\lambda c-a)(\lambda-b) \lambda}{(\mu-1)^{2}} \\
\frac{-(\lambda-b)^{2} \lambda}{\mu-1} & \frac{-(\mu a-\lambda c-a)(\lambda-b) \lambda}{(\mu-1)^{2}} & \frac{(\lambda-b)^{2}}{(\mu-1)^{2}}
\end{array}\right),
$$

Note that $\mu \neq 1$, as $\operatorname{det} \eta^{2} \neq 0$.

The WDVV system in first-order form also admits a non-local Hamiltonian operator of Ferapontov type $A_{1}^{2}$ that is compatible with $A_{2}^{2}$. We present the special case where $\mu=2$, as the general case has an expression that is too big to be shown here. We have

$$
g^{i j}=\left(\begin{array}{ccc}
2 b \lambda-a^{2} \lambda-b^{2}-5 & a-a b \lambda-b c+c \lambda & 2 b-b^{2} \lambda-c^{2}-2 \lambda \\
a-a b \lambda-b c+c \lambda & 2 b-b^{2} \lambda-c^{2}-2 \lambda & \frac{c\left(a c \lambda-2 b^{2} \lambda+4 b-c^{2}-\lambda\right)}{b-\lambda} \\
2 b-b^{2} \lambda-c^{2}-2 \lambda & \frac{c\left(a c \lambda-2 b^{2} \lambda+4 b-c^{2}-\lambda\right)}{b-\lambda} & \frac{\delta}{(b-\lambda)^{2}},
\end{array}\right)
$$

where

$$
\delta=\lambda\left(b^{2}\left(4 b-3 c^{2}\right)-2 a c\left(2 b-c^{2}\right)+8 b-c^{2}\right)-\left(4 b-c^{2}\right)\left(2 b-c^{2}\right)-4-\left(a c-b^{2}\right)^{2}
$$

and the value of the constants in the non-local part is $\alpha=1, \beta=0, \gamma=\lambda$.

The case $\eta^{3}$. In this case we have

$$
\phi(a, b, c)=\frac{b^{2}-a c+b c-2 b+1}{a} .
$$

There exists a unique third-order HHO $A_{2}^{3}$, it is generated by the following metric of type $g^{(4)}[33,34]$ :

$$
h_{i j}=\left(\begin{array}{ccc}
(1-b-c)(b+c-3) & a(b+c-2)+1 & a(b+c-2) \\
a(b+c-2)+1 & -a^{2} & -a^{2} \\
a(b+c-2) & -a^{2} & -a^{2}
\end{array}\right) .
$$


We also have an operator $A_{1}^{3}$ of Ferapontov type (1.18). It is determined by the metric

$$
g^{i j}=\left(\begin{array}{ccc}
-a^{2}-2 a b & 2 a-a b-a c-b^{2} & 4 b-b^{2}-2 b c-3 \\
2 a-a b-a c-b^{2} & 4 b-b^{2}-2 b c-3 & \frac{4 a c-a c^{2}-b^{3}-b^{2} c+2 b^{2}-b}{a} \\
4 b-b^{2}-2 b c-3 & \frac{4 a c-a c^{2}-b^{3}-b^{2} c+2 b^{2}-b}{a} & \frac{\delta}{a}
\end{array}\right),
$$

where $\delta=a c^{2}-4 a c-2 b^{2} c+4 b^{2}-2 b c^{2}+8 b c-8 b-2 c+4$. The nonlocal part of the operator is determined by $\alpha=0, \beta=1, \gamma=1$.

Furthermore, the Schouten bracket vanishes: $\left[A_{1}^{3}, A_{2}^{3}\right]=0$.

The case $\boldsymbol{\eta}^{4}$. In this case the function $\phi(a, b, c)$

$$
\phi(a, b, c)=\frac{\mu b^{2}-\mu a c+\lambda c^{2}-1}{\lambda b} .
$$

There exists a third-order HHO which is in the class $g^{(3)}[33,34]$. It is determined by the metric

$$
h_{i j}=\left(\begin{array}{ccc}
b^{2}+\mu & b \mu(\lambda c-\mu a) & -\mu \lambda b^{2} \\
b \mu(\lambda c-\mu a) & \lambda+a^{2}-\lambda c(2 \mu a-\lambda c) & \lambda b(\mu a-\lambda c) \\
-\mu \lambda b^{2} & \lambda b(\mu a-\lambda c)) & b^{2}
\end{array}\right) .
$$

Below we provide the metric $g^{i j}$ that defines $A_{1}^{4}$ :

$$
g^{i j}=\frac{\lambda}{\mu}\left(\begin{array}{ccc}
-a^{2} \mu-b^{2} \lambda-4 \mu \lambda & -b(a \mu+c \lambda) & -b^{2} \mu-c^{2} \lambda-1 \\
-b(a \mu+c \lambda) & -b^{2} \mu-c^{2} \lambda-1 & \frac{c\left(a c \mu-2 b^{2} \mu-c^{2} \lambda+1\right)}{b} \\
-b^{2} \mu-c^{2} \lambda-1 & \frac{c\left(a c \mu-2 b^{2} \mu-c^{2} \lambda+1\right)}{b} & \frac{\delta}{b^{2}}
\end{array}\right),
$$

where $\delta=2 a b^{2} c \lambda-a^{2} c^{2} \lambda+2 a c^{3} \mu-2 a c \mu \lambda-b^{4} \lambda-3 b^{2} c^{2} \mu-2 b^{2} \mu \lambda-c^{4} \lambda+2 c^{2}-\lambda$ and the values of constants in (1.18) are $\alpha=\mu, \beta=0, \gamma=\lambda$. Also in this case $\left[A_{1}^{4}, A_{2}^{4}\right]=0$ for any $\lambda, \mu= \pm 1$.

\subsection{An example: equation of flat centroaffine metrics}

There is another significant example provided in [24] in the case $N=3$ :

$$
\eta=\left(\begin{array}{lll}
1 & 0 & 0 \\
0 & 0 & 1 \\
0 & 1 & 0
\end{array}\right)
$$

This particular example has an interesting geometric interpretation as the equation of flat centroaffine metrics for surfaces in $\mathbb{R}^{3}[28]$. The WDVV equation takes the form

$$
f_{x x x} f_{y y y}-f_{x x y} f_{x y y}=1,
$$

and the system in first-order form reads:

$$
\begin{aligned}
a_{t} & =b_{x}, \\
b_{t} & =c_{x}, \\
c_{t} & =\left(\frac{b c+1}{a}\right)_{x} .
\end{aligned}
$$


The above system admits a third-order HHO of type $g^{(4)}$ which is again completely determined by a metric $h_{i j}$ :

$$
h_{i j}=\left(\begin{array}{ccc}
c^{2} & -1 & -a c \\
-1 & 0 & 0 \\
-a c & 0 & a^{2}
\end{array}\right)
$$

and has the form:

$$
A_{2}=\left(\begin{array}{ccc}
0 & -\partial_{x}^{3} & 0 \\
-\partial_{x}^{3} & 0 & -\partial_{x}^{2} \frac{c}{a} \partial_{x} \\
0 & -\partial_{x} \frac{c}{a} \partial_{x}^{2} & \frac{1}{2}\left(\partial_{x}^{2} \frac{1}{a^{2}} \partial_{x}+\partial_{x} \frac{1}{a^{2}} \partial_{x}^{2}\right)
\end{array}\right) .
$$

For this case we also have a first-order nonlocal operator $A_{1}$ which is determined by metric $g^{i j}$ :

$$
g^{i j}=\left(\begin{array}{ccc}
2 a b & a c+b^{2} & 2 b c+3 \\
a c+b^{2} & 2 b c+3 & \frac{a c^{2}+b^{2} c+b}{a} \\
2 b c+3 & \frac{a c^{2}+b^{2} c+b}{a} & \frac{2 c(b c+1)}{a}
\end{array}\right),
$$

and its tail vector $\alpha=\gamma=0, \beta=-1$. The operator is compatible with $A_{2}:\left[A_{1}, A_{2}\right]=0$. This turns the equation of flat centroaffine metrics, in the form of a first-order quasilinear system of PDEs (4.25), into a bi-Hamiltonian system.

Example 3 in [34] is just another form of (4.24), after transforming $\eta^{(2)}$ into the identity matrix $(\mu=1)$. Also this case is bi-Hamiltonian by means of the third-order HHO in Example 3 and the first-order nonlocal operator presented in [14].

Remark 7. Ferapontov operators of type (1.18) can be transformed into local operators by a reciprocal transformation if $\alpha=\gamma=0$ [32]. The fact that in the above case the first-order operator is localizable does not contradict the fact that, according to [51], it is not localizable by equivalence transformations of WDVV. Indeed, in [30] a transformation between (1.7) and the above (4.24) which can also be read as a reciprocal transformation between the first-order systems is provided. It should not be a difficult exercise to show that such a transformation brings the local first-order HHO of (1.7) into the above non-local first-order HHO.

\subsection{Remarks on the generic case}

A computation in the generic case is possible. In particular, if $N=3$ a generic choice of the matrix $\eta$ leads to a first-order system of the form (3.8). We can prove (by computer) that if $\eta_{11} \neq 0$ the generic metric of the third-order operator is of type $g^{(3)}$, while if $\eta_{11}=0$ it is of type $g^{(4)}$ (see [33]). Such computations show that, at least in the case $N=3$, the third-order operators exist independently, being the first-order system generated by the algorithm or as a relation between the coordinates $\mathrm{f}$ involving the WDVV equation. This fact suggests that a natural framework for characterizing the existence of a third-order HHO might be that of [2], where all conserved densities of the first-order system (in our case 5) are used to identify the system with a projective variety.

It is interesting to observe that our examples do not cover the whole range of Monge metrics that are classified in [33], as the most generic types $g^{(1)}$ and $g^{(2)}$. However, strictly 
speaking an occurrence of a metric of the latter classes in first-order WDVV systems cannot be excluded.

It is interesting to observe that, when $N=3$, the first-order WDVV systems produced by the Algorithm are always of the reducible type (3.10), according with the terminology in [1]. In the same paper it is proved that linearly degenerate reduced systems are semiHamiltonian. In our case we proved that such systems admit a third-order HHO, and hence they are linearly degenerate (see [35] and section 6), but we prove that the systems are bi-Hamiltonian (which, of course, imply semi-Hamiltonianity).

\section{WDVV equations in higher dimensions}

We still have no results of invariance of the bi-Hamiltonian structure for higher dimensions. However, there are strong indications that third-order HHOs exist for both normal forms $\eta^{(1)}$ and $\eta^{(2)}$ and all values of $N$, due to their existence for $N=4$ and $N=5$. In one case, the third-order HHO is paired with a first-order local HHO and we have a bi-Hamiltonian pair. We present the main results in this section.

We shall remark that at the moment there exists only one first-order operator for $N=4$ and $\eta=\eta^{(1)}$ : it was found in [31]. For $N=4$ and $\eta=\eta^{(2)}$ and $N=5$ we cannot exhibit any first-order operator (local or non-local) because the properties that we used in the case $N=3$ [11] do not hold in higher dimensions. It is however reasonable to conjecture that we will continue to have first-order operators, local in the case $\eta=\eta^{(1)}$ and nonlocal of type (1.18) in the case $\eta=\eta^{(2)}$, and that they will form bi-Hamiltonian pairs with the corresponding (conjectural) third-order operators.

\subsection{The case $N=4$}

In the case $N=4$ for long time only a first-order local HHO was known [31] in the case $\eta^{(1)}$ (1.14). A few years ago, a third-order HHO compatible with the known first-order HHO was found by a complicated procedure [55], later simplified in [35] by means of equations (2.6).

Here we will present a third-order HHO for Dubrovin's normal form $\eta^{(2)}$. This particular form of $\eta$ generates the following WDVV system (here $(t, x, y, z)=\left(t^{1}, t^{2}, t^{3}, t^{4}\right)$ ):

$$
\begin{aligned}
\mu f_{y y z} f_{z z z}+2 f_{y y z} f_{x y z}-f_{y y y} f_{x z z}-f_{x y y} f_{y z z}-\mu f_{y z z}^{2} & =0, \\
f_{x x y} f_{y z z}-f_{x x z} f_{y y z}-\mu f_{z z z} f_{x y z}+f_{z z z}+f_{x y y} f_{x z z}+\mu f_{x z z} f_{y z z}-f_{x y z}^{2} & =0, \\
f_{x x y} f_{y y z}-f_{x x z} f_{y y y}+\mu f_{y y z} f_{x z z}-\mu f_{x y z} f_{y z z}+f_{y z z} & =0, \\
f_{x x y} f_{x z z}-\mu f_{x x z} f_{z z z}-2 f_{x x z} f_{x y z}+f_{x x x} f_{y z z}+\mu f_{x z z}^{2} & =0, \\
f_{x x z} f_{x y y}+\mu f_{x x z} f_{y z z}-f_{y y z} f_{x x x}-\mu f_{x z z} f_{x y z}+f_{x z z} & =0, \\
f_{x x y} f_{x y y}+\mu f_{x x z} f_{y y z}-f_{x x x} f_{y y y}-\mu f_{x y z}^{2}+2 f_{x y z} & =0 .
\end{aligned}
$$

The above overdetermined system of non-linear PDEs can be rewritten in the form of two commuting hydrodynamic-type system using the identifications $a=f_{x x x}, b=f_{x x y}, c=$ 
$f_{x x z}, d=f_{x y y}, e=f_{x y z}, f=f_{x z z}$. One of the systems is:

$$
\begin{aligned}
a_{y}= & b_{x}, \quad b_{y}=d_{x}, \quad c_{y}=e_{x}, \\
d_{y}= & \left(\frac{b c d f \mu^{2}-b c e^{2} \mu^{2}-c^{2} d e \mu^{2}+a e^{3} \mu^{2}+b^{2} c d \mu-}{\delta}\right. \\
& \left.+\frac{-b a d e \mu+2 b c e \mu+c^{2} d \mu-3 a e^{2} \mu+b a d+c f \mu+2 a e}{\delta}\right)_{x}, \\
e_{y}= & \left(\frac{-c^{2} f e \mu^{2}+a f^{2} e \mu^{2}+b a f e \mu+c^{3} d \mu-}{\delta}\right. \\
& \left.+\frac{-c a d f \mu-c a e^{2} \mu+c^{2} f \mu-a f^{2} \mu-b a f+2 c a e}{\delta}\right)_{x}, \\
f_{y}= & \left(\frac{-c^{2} e^{2} \mu^{2}+a f e^{2} \mu^{2}+b c^{2} d \mu-c a d e \mu+2 c^{2} e \mu-2 a f e \mu+c a d+a f}{\delta}\right)_{x},
\end{aligned}
$$

where $\delta=-c^{3} \mu^{2}+c a f \mu^{2}+b c a \mu-a^{2} e \mu+a^{2}$. As an example, the right-hand side of the fourth equation comes from the compatibility condition $d_{y}=\left(f_{y y y}\right)_{x}$ and the expression of $f_{y y y}$ in terms of $a, \ldots, f$ through the system (5.1).

The above quasilinear system of first-order PDEs admits a unique third-order HHO determined by the following Monge metric $h_{i j}$ through the equations (2.6) (only entries with $i<j$ are shown):

$$
\begin{array}{rlrl}
h_{11} & =d^{2} & h_{12} & =e^{2} \mu-2 e \\
h_{13} & =2 d(-e \mu+1) & h_{14} & =-a d+c e \mu-c \\
h_{15} & =\mu(b / \mu-b e+c d-e f \mu+f) & h_{16} & =e^{2} \mu^{2}-2 e \mu+1 \\
h_{22} & =2 c(e \mu-1) & h_{23} & =-b e \mu+b-c d \mu-e f \mu^{2}+f \mu \\
h_{24} & =c^{2} \mu & h_{25} & =-a e \mu+a-b c \mu-c f \mu^{2} \\
h_{26} & =2 c \mu(e \mu-1) & h_{33} & =2 \mu\left(b d+d f \mu+e^{2} \mu / 2-e+1 / \mu\right) \\
h_{34} & =a e \mu-a-b c \mu-c f \mu^{2} & h_{35} & =\mu\left(a d+b^{2}+2 b f \mu-c e \mu+c+f^{2} \mu^{2}\right) \\
h_{36} & =\mu\left(b-b e \mu-c d \mu-e f \mu^{2}+f \mu\right) & h_{44} & =a^{2} \\
h_{45} & =-2 a c \mu & h_{46} & =c^{2} \mu^{2} \\
h_{55} & =\mu\left(2 a b+2 a f \mu+c^{2} \mu\right) & h_{56} & =\mu\left(-a e \mu+a-b c \mu-c f \mu^{2}\right) \\
h_{66} & =2 c \mu^{2}(e \mu-1) &
\end{array}
$$

Remark 8. We have 2 commuting quasilinear first-order systems of PDEs for each choice of $\eta$, and it is not automatically true that if one of them is bi-Hamiltonian the other will be bi-Hamiltonian with respect to the same operators. However, this is true in the case $\eta^{(1)}$ and $N=4$ [55]. More generally, it is known [63, 64] that if a diagonalizable quasilinear first-order system of PDEs is Hamiltonian with respect to a first-order HHO, then other commuting diagonalizable systems will be Hamiltonian with respect to the same operator. Even if this statement carries on to our non-diagonalizable first-order WDVV systems 
(see section 6), at the moment we can only conjecture that this extends to the compatible third-order operators.

\subsection{The case $N=5$}

This case is completely open: no Hamiltonian formulation was known until now. We have been able to find one new third-order Hamiltonian operator for the normal form $\eta^{(1)}(1.14)$. The first-order WDVV systems are 10-component systems; one of them (found using $t^{2}$ and $t^{3}$ in the Algorithm) admits one third-order HHO (up to a constant factor) that is defined by the following Monge metric $h_{i j}$ (only nonzero entries with $i<j$ are shown):

$$
\begin{array}{ll}
h_{11}=-u^{7} & h_{12}=-2 u^{6} u^{7} \\
h_{13}=\left(u^{6}\right)^{2}+2 u^{9} & h_{14}=-2 u^{7} \\
h_{15}=-\left(u^{6}\right)^{2} & h_{16}=u^{2} u^{7}-u^{3} u^{6}+u^{5} u^{6}+u^{8} \\
h_{17}=u^{1} u^{7}+u^{2} u^{6}+u^{4} & h_{18}=-2 u^{6} \\
h_{19}=-u^{3} & h_{110}=-1 \\
h_{22}=2 u^{3} u^{7}-2 u^{5} u^{7}-\left(u^{6}\right)^{2}+2 u^{9} & h_{23}=-u^{2} u^{7}-u^{3} u^{6}+u^{5} u^{6}+u^{8} \\
h_{24}=-2 u^{6} & h_{25}=u^{2} u^{7}+u^{3} u^{6}-u^{5} u^{6}+u^{8} \\
h_{26}=u^{1} u^{7}+u^{2} u^{6}+\left(u^{3}-u^{5}\right)^{2}+u^{4} & h_{27}=u^{1} u^{6}-u^{2} u^{3}+u^{2} u^{5} \\
h_{28}=u^{3}-2 u^{5} & h_{29}=-u^{2} \\
h_{33}=2 u^{2} u^{6}+2 u^{4} & h_{34}=-u^{3} \\
h_{35}=-2 u^{2} u^{6} & h_{36}=-u^{1} u^{6}-u^{2} u^{3}+u^{2} u^{5} \\
h_{37}=\left(u^{2}\right)^{2} & h_{38}=-2 u^{2} \\
h_{39}=-u^{1} & h_{44}=-2 \\
h_{46}=u^{2} & h_{47}=u^{1} \\
h_{55}=2 u^{2} u^{6} & h_{56}=u^{1} u^{6}+u^{2} u^{3}-u^{2} u^{5} \\
h_{57}=-\left(u^{2}\right)^{2} & h_{58}=u^{2} \\
h_{66}=2 u^{1} u^{3}-2 u^{1} u^{5}-\left(u^{2}\right)^{2} & h_{67}=-2 u^{1} u^{2} \\
h_{68}=u^{1} & h_{77}=-\left(u^{1}\right)^{2}
\end{array}
$$

For the second normal form $\eta^{(2)}$ we were able to find a third order operator as well, with the simplifying assumption $\mu=1$ (again, we used $t^{2}$ and $t^{3}$ in the Algorithm). Indeed, the computation is too hard for the servers that we can use if we allow $\mu$ to be a generic non-zero constant. The operator is defined by the following Monge metric $h_{i j}$ (again, we present only non-zero entries with $i \leq j$ ):

$$
\begin{array}{ll}
h_{11}=\left(u^{7}\right)^{2} & h_{12}=2 u^{7} u^{6} \\
h_{13}=-\left(u^{6}\right)^{2}+\left(u^{9}\right)^{2}-2 u^{9} & h_{14}=-\left(2 u^{9}-2\right) u^{7} \\
h_{15}=\left(u^{6}\right)^{2} & h_{16}=-u^{2} u^{7}+u^{3} u^{6}-u^{5} u^{6}+u^{8} u^{9}-u^{8} \\
h_{17}=-u^{1} u^{7}-u^{2} u^{6}+u^{4} u^{9}-u^{4} & h_{18}=-\left(2 u^{9}-2\right) u^{6}
\end{array}
$$




$$
\begin{aligned}
& h_{19}=-u^{10} u^{9}-u^{3} u^{9}+u^{4} u^{7}+u^{6} u^{8}+u^{10}+u^{3} \quad h_{110}=\left(u^{9}\right)^{2}-2 u^{9}+1 \\
& h_{22}=-2 u^{3} u^{7}+2 u^{5} u^{7}+\left(u^{6}\right)^{2}+\left(u^{9}\right)^{2}-2 u^{9} \quad h_{23}=u^{2} u^{7}+u^{3} u^{6}-u^{5} u^{6}+u^{8} u^{9}-u^{8} \\
& h_{24}=-2 u^{6} u^{9}-2 u^{7} u^{8}+2 u^{6} \quad h_{25}=-u^{2} u^{7}-u^{3} u^{6}+u^{5} u^{6}+u^{8} u^{9}-u^{8} \\
& h_{26}=-u^{1} u^{7}-u^{2} u^{6}-\left(u^{3}\right)^{2}+2 u^{3} u^{5}+\quad h_{27}=-u^{1} u^{6}+u^{2} u^{3}-u^{2} u^{5}+u^{4} u^{8} \\
& +u^{4} u^{9}-\left(u^{5}\right)^{2}+\left(u^{8}\right)^{2}-u^{4} \\
& h_{28}=u^{9}\left(u^{3}+u^{10}-2 u^{5}\right)+u^{4} u^{7}-u^{6} u^{8} \quad h_{29}=u^{8}\left(u^{5}-u^{10}-2 u^{3}\right)-u^{2} u^{9}+u^{4} u^{6}+u^{2} \\
& +u^{10}-u^{3}+2 u^{5} \\
& h_{210}=\left(2 u^{9}-2\right) u^{8} \quad h_{33}=-2 u^{2} u^{6}+2 u^{4} u^{9}-2 u^{4} \\
& h_{34}=-u^{10} u^{9}-u^{3} u^{9}-u^{4} u^{7}+u^{6} u^{8}+u^{10}+u^{3} \quad h_{35}=2 u^{6} u^{2} \\
& h_{36}=u^{1} u^{6}+u^{2} u^{3}-u^{2} u^{5}+u^{4} u^{8} \quad h_{37}=-\left(u^{2}\right)^{2}+\left(u^{4}\right)^{2} \\
& h_{38}=-2 u^{2} u^{9}-2 u^{4} u^{6}+2 u^{2} \quad h_{39}=-u^{1} u^{9}-u^{10} u^{4}+u^{2} u^{8}-u^{3} u^{4}+u^{1} \\
& h_{310}=\left(2 u^{9}-2\right) u^{4} \quad h_{44}=2 u^{10} u^{7}+2 u^{3} u^{7}+\left(u^{6}\right)^{2}+\left(u^{9}\right)^{2}-2 u^{9}+2 \\
& h_{45}=-2 u^{8} u^{6} \quad h_{46}=-u^{10} u^{8}+u^{2} u^{9}-2 u^{3} u^{8}-u^{4} u^{6}+u^{5} u^{8}-u^{2} \\
& h_{47}=u^{1} u^{9}-u^{10} u^{4}+u^{2} u^{8}-u^{3} u^{4}-u^{1} \quad h_{48}=2 u^{10} u^{6}+u^{2} u^{7}+u^{3} u^{6}+u^{5} u^{6}+u^{8} u^{9}-u^{8} \\
& h_{49}=u^{1} u^{7}+\left(u^{10}\right)^{2}+2 u^{10} u^{3}+u^{2} u^{6}+\quad h_{410}=-u^{10} u^{9}-u^{3} u^{9}-u^{4} u^{7}-u^{6} u^{8}+u^{10}+u^{3} \\
& +\left(u^{3}\right)^{2}-u^{4} u^{9}-\left(u^{8}\right)^{2}+u^{4} \\
& h_{55}=-2 u^{2} u^{6}+\left(u^{8}\right)^{2} \quad h_{56}=-u^{1} u^{6}-u^{2} u^{3}+u^{2} u^{5}+u^{4} u^{8} \\
& h_{57}=\left(u^{2}\right)^{2} \quad h_{58}=-u^{10} u^{8}+u^{2} u^{9}+u^{4} u^{6}-u^{5} u^{8}-u^{2} \\
& h_{59}=-2 u^{8} u^{2} \quad h_{510}=\left(u^{8}\right)^{2} \\
& h_{66}=-2 u^{1} u^{3}+2 u^{1} u^{5}+\left(u^{2}\right)^{2}+\left(u^{4}\right)^{2} \quad h_{67}=2 u^{2} u^{1} \\
& h_{68}=u^{1} u^{9}-u^{10} u^{4}-u^{2} u^{8}+u^{3} u^{4}-2 u^{4} u^{5}-u^{1} h_{69}=-2 u^{1} u^{8}-2 u^{2} u^{4} \\
& h_{610}=2 u^{8} u^{4} \quad h_{77}=\left(u^{2}\right)^{2} \\
& h_{78}=-2 u^{4} u^{2} \quad h_{79}=-2 u^{4} u^{1} \\
& h_{710}=\left(u^{4}\right)^{2} \\
& h_{89}=u^{1} u^{6}+2 u^{10} u^{2}+u^{2} u^{3}+u^{2} u^{5}+u^{4} u^{8} \quad h_{810}=-u^{10} u^{8}-u^{2} u^{9}-u^{4} u^{6}-u^{5} u^{8}+u^{2} \\
& h_{99}=2 u^{1} u^{10}+2 u^{1} u^{3}+\left(u^{2}\right)^{2}+\left(u^{4}\right)^{2} \quad h_{910}=-u^{1} u^{9}-u^{10} u^{4}-u^{2} u^{8}-u^{3} u^{4}+u^{1} \\
& h_{1010}=2 u^{4} u^{9}+\left(u^{8}\right)^{2}-2 u^{4}
\end{aligned}
$$

\section{Conclusions: projective geometry of WDVV systems}

The results that we achieved so far have very interesting implications in terms of projective geometry that we will discuss in this section (see also the Introduction).

In a recent paper [35] it was proved that any hydrodynamic-type system that has a third-order homogeneous Hamiltonian operator has a rich geometric structure. The results transfer to first-order WDVV systems. Indeed, in the case $N=3$ we have:

1. There exists a quadratic line complex of lines in the projective space $\mathbb{P}^{3}$ associated with the first-order WDVV system. The construction is a straightforward generalization of what we have written in the Introduction. 
2. The first-order WDVV system defines a linear line congruence in the projective space $\mathbb{P}^{4}$. This is a 3 -parameter family of lines in the projective space $\mathbb{P}^{4}$. Again, this is shown in the Introduction.

3. The first-order WDVV system is linearly degenerate and belongs to the Temple class.

4. The first-order WDVV system is non-diagonalizable.

5. The first-order WDVV system admits a Hamiltonian and a momentum with respect to the third-order HHO; their expressions are local after a potential substitution $b_{x}^{i}=u^{i}$ and are given by explicit formulae [35].

6. The first-order WDVV systems are equivalent to the system (1.8) using a projective reciprocal transformation of the type (1.26).

Let $N=4,5$. Then, the above properties (with the exception of equivalence) hold for the first-order WDVV systems that we considered in section 5 for the normal forms $\eta^{(1)}, \eta^{(2)}$.

Several considerations can be made in view of future research.

Conjecture: every WDVV system is associated with a quadratic line complex and a linear line congruence. This is just a rephrasing of the conjecture that every first-order WDVV system admits a local third-order HHO. However, the Conjecture put in this way suggests that WDVV equations have a projective-geometric interpretation that is yet to be uncovered. The generalization to the Oriented Associativity equations for Fmanifolds is an active research topic (see e.g. [6]) and quadratic line complexes occur also in that case [56] (see also [53]). A future role of such objects besides the bi-Hamiltonian structure that they provide is foreseeable.

Projective equivalence of WDVV systems. The last statement in the above list implies that, in the case $N=3$, if our WDVV systems admit a third-order homogeneous operator they should in principle be all equivalent. In practice, it is extremely difficult to solve the equations for the unknown transformation. An explicit transformation between the WDVV equations (1.7) and (4.24) in [31] shows the computational difficulties. It is preferable to recompute the operators for any specific presentation of the WDVV system. Note that the transformation (1.26) is not an invariance transformation of WDVV equations. In principle, the equivalence does no longer hold in higher dimensions.

First-order operators and projective geometry. First-order local or non-local HHOs do not have a projective-geometric interpretation yet. However, we expect that those that are compatible with third-order HHOs will have a projective-geometric role. It is an interesting remark the fact that the metric of the first-order operators that we found have rational coefficients (in upper indices!) and the denominator is always the square root of the determinant of the Monge metric. Such a determinant is a perfect square, and its zero locus is the Kümmer surface of the underlying quadratic line complex, see [33, 34]. 
Projective geometry in the original WDVV setting. The projective-geometric structures that we found so far might be transported in the 'initial' setting of WDVV. A theoretical framework for the Hamiltonian formalism for general PDEs has already been developed in [44]. In that framework, variational bivectors for the WDVV equation in the non-evolutionary form (1.7) have been found (although with an explicit dependence on the independent variables) [45]. An understanding of the role of quadratic line complexes and line congruences in the initial formulation might shed new light on the relationship between WDVV equations and projective-geometric invariants.

\section{Acknowledgments}

R.V. would like to thank M. Casati, E.V. Ferapontov, J.S. Krasil'shchik, P. Lorenzoni, M.V. Pavlov, A. Sergyeyev, A.M. Verbovetsky for many scientific discussions throughout years of scientific cooperation. Thanks are also due to M. Beccaria for useful suggestions.

Computational resources were supplied by the project "e-Infrastruktura CZ" (e-INFRA LM2018140) provided within the program Projects of Large Research, Development and Innovations Infrastructures and by the Dept. of Mathematics and Physics "E. De Giorgi" of the Università del Salento.

The research of JV was supported in part by the Specific Research grant SGS/13/2020 of the Silesian University in Opava, Czechia.

The research of RV has been funded by the Dept. of Mathematics and Physics "E. De Giorgi" of the Università del Salento, Istituto Naz. di Fisica Nucleare IS-CSN4 Mathematical Methods of Nonlinear Physics, GNFM of Istituto Nazionale di Alta Matematica.

Open Access. This article is distributed under the terms of the Creative Commons Attribution License (CC-BY 4.0), which permits any use, distribution and reproduction in any medium, provided the original author(s) and source are credited.

\section{References}

[1] S. Agafonov, Linearly degenerate reducible systems of hydrodynamic type, J. Math. Anal. Appl. 222 (1998) 15.

[2] S.I. Agafonov, Duality for systems of conservation laws, Lett. Math. Phys. 110 (2019) 1123.

[3] S.I. Agafonov and E.V. Ferapontov, Systems of conservation laws from the point of view of the projective theory of congruences, Izv. Akad. Nauk SSSR Ser. Mat. 60 (1996) 3.

[4] S.I. Agafonov and E.V. Ferapontov, Systems of conservation laws of Temple class, equations of associativity and linear congruences in $\mathbb{P}^{4}$, Manuscripta Math. 106 (2001) 461 [math/0106028].

[5] G. Antoniou and M. Feigin, Supersymmetric V-systems, JHEP 02 (2019) 115 [arXiv: 1812.02643] [INSPIRE].

[6] A. Arsie, A. Buryak, P. Lorenzoni and P. Rossi, Semisimple flat F-manifolds in higher genus, arXiv: 2001.05599 [INSPIRE]. 
[7] A.V. Balandin and G.V. Potemin, On non-degenerate differential-geometric Poisson brackets of third order, Russ. Math. Surv. 56 (2001) 976.

[8] H. Baran and M. Marvan, Jets. A software for differential calculus on jet spaces and diffieties, Silesian University in Opava, Opava Czechia, 2003 and 2010, http://jets.math.slu.cz/.

[9] A. Basalaev, P. Dunin-Barkowski and S. Natanzon, Integrable hierarchies associated to infinite families of Frobenius manifolds, J. Phys. A 54 (2021) 115201 [InSPIRE].

[10] A.A. Belavin and V.A. Belavin, Frobenius manifolds, Integrable Hierarchies and Minimal Liouville Gravity, JHEP 09 (2014) 151 [arXiv:1406.6661] [INSPIRE].

[11] O.I. Bogoyavlenskij, Necessary conditions for existence of non-degenerate Hamiltonian structures, Commun. Math. Phys. 182 (1996) 253.

[12] Y. Cao and Y. Toda, Gopakumar-Vafa Type Invariants on Calabi-Yau 4-Folds via Descendent Insertions, Commun. Math. Phys. 383 (2021) 281 [arXiv:2003.00787] [INSPIRE].

[13] M. Casati, E.V. Ferapontov, M.V. Pavlov and R.F. Vitolo, On a class of third-order nonlocal Hamiltonian operators, J. Geom. Phys. 138 (2019) 285.

[14] M. Casati, P. Lorenzoni, D. Valeri and R. Vitolo, Weakly nonlocal Poisson brackets: tools, examples, computations, arXiv:2101.06467.

[15] M. Casati, P. Lorenzoni and R. Vitolo, Three computational approaches to weakly nonlocal poisson brackets, Stud. Appl. Math. 144 (2020) 412 [arXiv:1903.08204].

[16] G. Cotti, Degenerate Riemann-Hilbert-Birkhoff problems, semisimplicity, and convergence of WDVV-potentials, arXiv:2011.04498.

[17] R. Dijkgraaf, H.L. Verlinde and E.P. Verlinde, Topological strings in $d<1$, Nucl. Phys. B 352 (1991) 59 [INSPIRE].

[18] X.-M. Ding, Y. Li and L. Meng, From r-Spin Intersection Numbers to Hodge Integrals, JHEP 01 (2016) 015 [arXiv: 1507.04093] [INSPIRE].

[19] I. Ya. Dorfman, Dirac Structures and Integrability of Nonlinear Evolution Equations, John Wiley \& Sons, New York U.S.A. (1993).

[20] P.W. Doyle, Differential geometric Poisson bivectors in one space variable, J. Math. Phys. 34 (1993) 1314.

[21] B. Dubrovin, Flat pencils of metrics and Frobenius manifolds, in Proceedings of 1997 Taniguchi Symposium "Integrable Systems and Algebraic Geometry", Kobe Japan (1997), M.-H. Saito, Y. Shimizu and K. Ueno eds., World Scientific, Sinagapore (1998), pg. 42, https://people.sissa.it/ dubrovin/bd_papers.html.

[22] B. Dubrovin and Y. Zhang, Normal forms of hierarchies of integrable PDEs, Frobenius manifolds and Gromov - Witten invariants, math/0108160 [INSPIRE].

[23] B.A. Dubrovin and S.P. Novikov, Poisson brackets of hydrodynamic type, Sov. Math. Dokl. 30 (1984) 651, http://www.mi-ras.ru/ snovikov/88.pdf.

[24] B. Dubrovin, Geometry of $2-D$ topological field theories, Lect. Notes Math. 1620 (1996) 120 [hep-th/9407018] [INSPIRE].

[25] B.A. Dubrovin, WDVV equations and Frobenius manifolds, in Encyclopedia of Mathematical Physics. Volume 1, Elsevier, Amsterdam The Netherlands (2006), pg. 438. 
[26] B.A. Dubrovin and S.P. Novikov, Hamiltonian formalism of one-dimensional systems of hydrodynamic type and the Bogolyubov-Whitham averaging method, Sov. Math. Dokl. 27 (1983) 665, http://www.mi-ras.ru/ snovikov/80.pdf.

[27] E.V. Ferapontov, Nonlocal Hamiltonian operators of hydrodynamic type: Differential geometry and applications, Am. Math. Soc. Transl. 170 (1995) 33.

[28] E.V. Ferapontov, Hypersurfaces with flat centroaffine metric and equations of associativity, Geom. Dedicata 103 (2004) 33 [math/0205248].

[29] E.V. Ferapontov, C.A.P. Galvao, O. Mokhov and Y. Nutku, Bi-Hamiltonian structure of equations of associativity in 2-d topological field theory, Commun. Math. Phys. 186 (1997) 649.

[30] O.I. Mokhov and E.V. Ferapontov, Equations of associativity in two-dimensional topological field theory as integrable Hamiltonian nondiagonalizable systems of hydrodynamic type, hep-th/9505180 [INSPIRE].

[31] E.V. Ferapontov and O.I. Mokhov, On the Hamiltonian representation of the associativity equations, in Algebraic aspects of integrable systems: in memory of Irene Dorfman, I.M. Gelfand and A.S. Fokas eds., Birkhäuser, Boston U.S.A. (1996), pg. 75.

[32] E.V. Ferapontov and M.V. Pavlov, Reciprocal transformations of Hamiltonian operators of hydrodynamic type: nonlocal Hamiltonian formalism for linearly degenerate systems, J. Math. Phys. 44 (2003) 1150 [nlin/0212026].

[33] E.V. Ferapontov, M.V. Pavlov and R.F. Vitolo, Projective-geometric aspects of homogeneous third-order Hamiltonian operators, J. Geom. Phys. 85 (2014) 16 [arXiv:1401.7235].

[34] E.V. Ferapontov, M.V. Pavlov and R.F. Vitolo, Towards the classification of homogeneous third-order Hamiltonian operators, Int. Math. Res. Notes 22 (2016) 6829 [arXiv: 1508.02752].

[35] E.V. Ferapontov, M.V. Pavlov and R.F. Vitolo, Systems of conservation laws with third-order Hamiltonian structures, Lett. Math. Phys. 108 (2018) 1525 [arXiv:1703.06173].

[36] E.V. Ferapontov, M.V. Pavlov, and L. Xue, Second-order integrable Lagrangians and WDVV equations, arXiv:2007.03768.

[37] A. Galajinsky and O. Lechtenfeld, Superconformal SU(1,1|n) mechanics, JHEP 09 (2016) 114 [arXiv: 1606.05230] [INSPIRE].

[38] I.M. Gel'fand and I. Ya. Dorfman, The Schouten bracket and Hamiltonian operators, Funct. Anal. Appl. 14 (1981) 223.

[39] O. Brauer and A. Buryak, Open topological recursion relations in genus 1 and integrable systems, JHEP 01 (2021) 048 [arXiv: 2008.06922] [INSPIRE].

[40] H. Jockers and P. Mayr, Quantum k-theory of Calabi-Yau Manifolds, JHEP 11 (2019) 011 [arXiv: 1905.03548] [INSPIRE].

[41] J. Kalayci and Y. Nutku, Bi-Hamiltonian structure of a WDVV equation in $2-D$ topological field theory, Phys. Lett. A 227 (1997) 177 [InSPIRE].

[42] J. Kalayci and Y. Nutku, Alternative biHamiltonian structures for WDVV equations of associativity, J. Phys. A 31 (1998) 723 [hep-th/9810076] [InSPIRE].

[43] P. Kersten, I. Krasilshchik and A. Verbovetsky, Hamiltonian operators and $\ell^{*}$-coverings, J. Geom. Phys. 50 (2004) 273 [math/0304245] [INSPIRE]. 
[44] P. Kersten, I. Krasil'shchik, A. Verbovetsky and R. Vitolo, Hamiltonian structures for general PDEs, in Differential equations: Geometry, Symmetries and Integrability. The Abel Symposium 2008, B. Kruglikov, V.V. Lychagin and E. Straume eds., Springer-Verlag, Berlin Germany (2009), pg. 187 [arXiv: 0812.4895].

[45] P. Kersten, I. Krasil'shchik, A. Verbovetsky and R. Vitolo, On integrable structures for a generalized Monge-Ampère equation, Theor. Math. Phys. 128 (2012) 600 [arXiv:1104.0258].

[46] N. Kozyrev, S. Krivonos, O. Lechtenfeld and A. Sutulin, SU(2|1) supersymmetric mechanics on curved spaces, JHEP 05 (2018) 175 [arXiv:1712.09898] [INSPIRE].

[47] J. Krasil'shchik, A. Verbovetsky and R. Vitolo, The symbolic computation of integrability structures for partial differential equations, Texts and Monographs in Symbolic Computation, Springer, NewYork U.S.A. (2018) [ISBN:978-3-319-71654-1], http://gdeq.org/Symbolic_Book.

[48] F. Magri, A Simple model of the integrable Hamiltonian equation, J. Math. Phys. 19 (1978) 1156 [INSPIRE].

[49] F. Magri and C. Morosi, A geometrical characterization of Hamiltonian systems through the theory of Poisson-Nijenhuis manifolds, Technical report, University of Milano-Bicocca, Milano Italy (2008), https://boa.unimib.it/handle/10281/17656?mode=simple.47.

[50] O. Mokhov, Symplectic and Poisson geometry on loop spaces of manifolds and nonlinear equations, hep-th/9503076 [INSPIRE].

[51] O.I. Mokhov and N.A. Pavlenko, Classification of the Associativity Equations with a First-Order Hamiltonian Operator, Theor. Math. Phys. 197 (2018) 1501 [InSPIRE].

[52] S.P. Novikov, S.V. Manakov, L.P. Pitaevskii and V.E. Zakharov, Theory of Solitons, Plenum Press, New York U.S.A. (1984).

[53] M. Pavlov and A. Sergyeyev, Oriented associativity equations and symmetry consistent conjugate curvilinear coordinate nets, J. Geom. Phys. 85 (2014) 46 [arXiv:1204.2514].

[54] M.V. Pavlov, Conservation of the "forms" of Hamiltonian structures upon linear substitution for independent variables, Math. Notes 57 (1995) 489.

[55] M.V. Pavlov and R.F. Vitolo, On the bi-Hamiltonian geometry of the WDVV equations, Lett. Math. Phys. 105 (2015) 1135 [arXiv:1409.7647].

[56] M.V. Pavlov and R.F. Vitolo, Bi-Hamiltonian structure of the Oriented Associativity equation, J. Phys. A 52 (2019) 20LT01 [arXiv:1812.01413].

[57] G.V. Potemin, Some aspects of differential geometry and algebraic geometry in the theory of solitons (in Russian), Ph.D. Thesis, Moscow State University, Moscow Russian Federation (1991).

[58] G.V. Potemin, On third-order Poisson brackets of differential geometry, Russ. Math. Surv. 52 (1997) 617.

[59] Y. Shen and J. Zhou, Ramanujan identities and quasi-modularity in Gromov-Witten theory, Commun. Number Theor. Phys. 11 (2017) 405.

[60] J.P. Solomon and S.B. Tukachinsky, Relative quantum cohomology, arXiv:1906.04795 [INSPIRE].

[61] R. Stedman and I.A.B. Strachan, Extended $\bigvee$-systems and trigonometric solutions to the WDVV equations, J. Math. Phys. 62 (2021) 022301 [INSPIRE]. 
[62] I.A.B. Strachan and Richard Stedman, Generalized Legendre transformations and symmetries of the WDVV equations, J. Phys. A 50 (2017) 095202.

[63] S. Tsarev, On Poisson brackets and one-dimensional Hamiltonian systems of hydrodynamic type, Sov. Math. Dokl. 31 (1985) 488.

[64] S.P. Tsarev, The geometry of Hamiltonian systems of hydrodynamic type. The generalized hodograph method, Math. USSR Izv. 37 (1991) 397.

[65] J. Vašíček and R. Vitolo, WDVV equations and invariant bi-Hamiltonian formalism: computer algebra programs,

GitHub:https://github.com/Jakub-Vasicek/WDVV-computations (2021).

[66] P. Vergallo and R. Vitolo, Homogeneous Hamiltonian operators and the theory of coverings, Diff. Geom. Appl. 75 (2021) 101713 [arXiv:2007.15294].

[67] R. Vitolo, Computing with Hamiltonian operators, Comput. Phys. Commun. 244 (2019) 228 [arXiv: 1808.03902].

[68] E. Witten, On the Structure of the Topological Phase of Two-dimensional Gravity, Nucl. Phys. B 340 (1990) 281 [INSPIRE].

[69] A. Zinger, Real Topological Recursions and WDVV Relations, arXiv:2003.05860 [INSPIRE]. 